\title{
Cross-Reactivity of Secondary Antibodies against African Rodents and Application for Sero-Surveillance
}

\author{
Ichiro NAKAMURA ${ }^{1,2) *}$, Bernard Mudenda HANG'OMBE ${ }^{2)}$, Hirofumi SAWA ${ }^{12}$ 2), Shintaro KOBAYASHI ${ }^{1)}$, \\ Yasuko ORBA ${ }^{1)}$, Akihiro ISHII ${ }^{1,2)}$, Yuka THOMAS ${ }^{1,2)}$, Rie ISOZUMI ${ }^{3)}$, Kumiko YOSHIMATSU ${ }^{3)}$, Aaron S. MWEENE ${ }^{2)}$, \\ Ayato TAKADA ${ }^{1,2)}$, Chihiro SUGIMOTO ${ }^{1,2)}$ and Jiro ARIKAWA ${ }^{3)}$ \\ 1)Hokkaido University Research Center for Zoonosis Control, N20W10, Kita-ku, Sapporo, Hokkaido 001-0020, Japan \\ ${ }^{2}$ School of Veterinary Medicine, University of Zambia, P. O. Box 32379, Lusaka, Zambia \\ ${ }^{3)}$ Graduate School of Medicine, Hokkaido University, N15W7, Kita-ku, Sapporo, Hokkaido 060-8638, Japan
}

(Received 26 October 2012/Accepted 23 January 2013/Published online in J-STAGE 6 February 2013)

ABSTRACT. A total of 466 rodents were captured in the Republic of Zambia from 2006 to 2010. Based on morphological observations and phylogenetic analyses of mitochondrial gene sequences, rodents were divided into 10 groups consisting of 39 Rattus rodents, 263 multimammate rats, 18 other Murinae rodents, 95 gerbils, 11 pouched mice, 1 giant-pouched rat, 38 fat mice and 1 dormouse. Rodent antibodies except that from Rattus were examined for their cross-reactivity to commercially available antibody detection reagents. Anti-mouse immunoglobulin $\mathrm{G}$ ( $\mathrm{IgG}$ ) was most cross-reactive to heterologous antibodies including multimammate rat, gerbil, pouched mouse and fat mouse. Thus, anti-mouse IgG would be a useful detection tool in serological examination of the Zambian rodent population. Preliminary sero-surveillance for plague, leptospirosis and hantavirus infection was performed by ELISA.

KEY WORDS: hantavirus, leptospirosis, plague, rodent-borne zoonoses, sero-surveillance.

doi: 10.1292/jvms.12-0471; J. Vet. Med. Sci. 75(6): 819-825, 2013

Rodents play a key role as reservoir hosts of causative agents of various bacterial, viral and parasitic zoonoses. They are considered to be an important reservoir, as they are often persistently infected and can transmit pathogens to humans. Consequently, surveillance of infected rodents provides crucial information for the prevention and control of rodent-borne zoonoses.

Serological examinations to detect antibodies against specific pathogens have been most widely used for surveillance. Indirect immunofluorescent antibody (IFA) assay, enzymelinked immunosorbent assay (ELISA) and Western blotting test are most commonly used in surveys, and they require species-specific secondary antibodies to the immunoglobulin (Ig) of the target animals. However, variable levels of cross-reactivity of the secondary antibodies against Ig of heterologous rodent species have been reported [12]. Therefore, specification of animal species and evaluation of crossreactivity of secondary antibodies used for the diagnoses of various animal species are essential to obtain proper results. Although local and sporadic cases of bubonic plague [6] and febrile illness with hemorrhage by unknown etiology have been reported, surveillance among wild animals, especially rodents, is quite limited in the Republic of Zambia. Although serological surveillance by convenient IFA test and ELISA is recommended, the absence of information regarding

\footnotetext{
*Correspondence to: Nakamura, I., Hokkaido University Research Center for Zoonosis Control, N20W10, Kita-ku, Sapporo, Hokkaido 001-0020, Japan.

e-mail: inaka@czc.hokudai.ac.jp

(C)2013 The Japanese Society of Veterinary Science
}

the cross-reactivity of commercially available secondary antibodies to Igs of indigenous rodents makes it difficult to obtain reliable information.

In the present study, the species of rodents captured in Zambia from 2006 to 2010 were classified by genetic characterization, and the levels of cross-reactivity of secondary antibodies to rodent sera were examined to evaluate their applicability to serological diagnoses. By using appropriate secondary antibodies, the prevalence of antibodies to typical rodent-borne pathogens, hantavirus, Yersinia pestis and Leptospira, was examined.

Wire-meshed cage traps $(145 \times 100 \times 230 \mathrm{~mm})$ and Sharman-type cage traps $(50 \times 65 \times 157 \mathrm{~mm})$ (Hoga-lab, Kyoto, Japan), baited with peanut butter-flavored maize meal supplemented with oat seeds, were used for animal trapping. Animals were treated in accordance with the guidelines of the Hokkaido University Animal Research Committee. Animals were anesthetized, and blood samples were collected by heart puncture. Animals were photographed, and their body sizes and weights were recorded. Serum samples were stored at $-30^{\circ} \mathrm{C}$. Diluted working stocks were inactivated at $55^{\circ} \mathrm{C}$ in a water bath for 30 min prior to use. Genomic DNA (gDNA) samples were extracted from livers using DNAzol ${ }^{\circledR}$ Reagent (Molecular Research Center, Inc., Cincinnati, OH, U.S.A.) according to the manufacture's protocol. Extracted DNA was reconstituted with $50 \mu l$ of Tris-EDTA buffer ( $\mathrm{pH}$ 8.0 ). For classification of host rodent species, a partial sequence of the cytochrome $c$ oxidase subunit I gene (COI) or cytochrome $b$ gene ( $c y t b)$ was amplified from liver gDNA samples. The primers used in this study are listed in Table 1. The amplicons were sequenced using an Applied Biosystem 3130xl Genetic Analyzer (Life Technologies Japan, 
Table 1. The oligonucleotide primers used in this study

\begin{tabular}{llll}
\hline Primer name & Target & \multicolumn{1}{c}{ Sequence $\left(5^{\prime}-3^{\prime}\right)$} & Referece \\
\hline L14724 & $c y t b$ & CGAAGCTTGATATGAAAAACCATCGTTG & {$[7]$} \\
L14723Sac & $c y t b$ & ACCATGACATGAAAAATCATCGTT & {$[3]$} \\
L15408Sac & $c y t b$ & ATAGACAAATCCCATTCCA & {$[3]$} \\
H15149 & $c y t b$ & CTGCAGCCCCTCAGAATGATATTTGTCCTC & This work \\
H15553Sac & $c y t b$ & ACCAGAATGATATTTCCTATTTGCCTA & {$[3]$} \\
H15554Den & $c y t b$ & CCAGAATGATATTTCTTATTCGCATAT & This work \\
H15915Sac & $c y t b$ & TCTCCATTTCTGGTTACAAGC & {$[3]$} \\
Rodcytb179R & $c y t b$ & GGATCGTTATGTAATGTGTAGGCTGTGAAA & This work \\
Rodcytb364F & $c y t b$ & GCAGTCATAGCAACCGCATTCATAGGTTAT & This work \\
Rodcytb782R & $c y t b$ & TCTGGGTCTGTTAATGTGTGGACGATTGGG & This work \\
L5310 & COI & CCTACTCAGCCATTTTACCTATG & {$[13]$} \\
L5579R & COI & ATTGGAGCCCCTGATATAGCATTCCCACGAATAAA & This work \\
R5709G & COI & GGGTAAACTGTTCAGCCTGTACCAGCCCCAGC & This work \\
R5712R & COI & GGTGGGTATACTGTTCATCCTGTTCCGGCTCC & This work \\
R5712M & COI & GGTGGGTAGACTGTTCAGCCGGTTCCTGCACC & This work \\
R6036R & COI & ACTTCTGGGTGTCCAAAGAATCA & {$[13]$} \\
\hline
\end{tabular}

Tokyo, Japan). All sequence data obtained in this study were deposited in the DNA Database of Japan (DDBJ) under the accession numbers AB672509-AB672521 and AB752503$\mathrm{AB} 753010$, and tentative names were given except for groups II, VII, VIII and X. Sequence data were aligned and analyzed by ClustalW2 program using neighbor-joining method with Kimura's parameter set (provided by European Bioinformatics Institute, http://www.ebi.ac.uk/Tools/msa/clustalw2/). Phylogenetic trees were visualized by the TreeView program ver. 1.6.6 (http://taxonomy.zoology.gla.ac.uk/rod/treeview. $\mathrm{html}$ ). The binding abilities of horseradish peroxidase (HRP) conjugates were examined by a method described previously [12] with some modifications. Two-fold serial dilutions of conjugates were tested against a constant volume of test serum samples. The recombinant $\mathrm{N}$-terminus 103 amino acid residues of nucleocapsid proteins (NP103) of Hantaan virus (HTNV) and of Puumala virus (PUUV) expressed in an Escherichia coli expression system [15, 16, 18] were used as ELISA antigens to detect hantavirus infection. In ELISA for plague and leptospirosis, recombinant Yersinia pestis F1 [2] and Leptospira LipL32 [4, 5] antigens expressed in E. coli were used, and the OD values against control antigens were subtracted from those of the test antigens to diminish the effect of variable background levels. Yersinia pestis F1 antigen was used as a control antigen for the detection of antibodies against the Leptospira LipL32 antigen, and Leptospira LipL32 antigen was used for the Yersinia pestis F1 antigen. Outliers of the subtractants examined by the Smirnov-Grubbs test (Ekuseru-Toukei 2008: Social Survey Research Information Co., Ltd., Tokyo, Japan) with a significance level at 0.01 were judged as positive.

From January 2006 to December 2010, a total of 466 rodents and 10 other animals were captured in two regions, Lusaka and Namwala, in the Republic of Zambia (Fig. 1). Two mitochondrial gene sequences, 702 base pairs (bp) of $C O I$ and the whole length of $c y t b$, were analyzed. From the 466 rodent genome samples, 411 sequences of COI (Fig. $2 \mathrm{~B})$ were obtained. Based on phylogenetic analysis of $\mathrm{COI}$ sequences, they were divided into 10 groups (groups I-X in Fig. 2A). While groups VII and VIII contained reference sequences of Rattus rattus and $R$. tanezumi, respectively, no available reference sequences were included in the other groups (Fig. 2A). A total of 171 sequences of $c y t b$ were further analyzed, and phylogenetic analysis of $c y t b$ sequences (Fig. 2C) showed that group I was a species of the fat mouse (Steatomys sp., subfamily Dendromurinae), group II was the pouched mouse (Saccostomus campestris, subfamily Cricetomyinae), group IV was a species of the multimammate rat (Mastomys sp., subfamily Murinae), group V was a species of the single-striped mouse (Lemniscomys sp., subfamily Murinae), group VII was the black rat ( $R$. rattus, subfamily Murinae) and group VIII was the tanezumi rat ( $R$. tanezumi, subfamily Murinae). Group IX was estimated to be a single species of the gerbil (subfamily Gerbillinae). Although the COI sequence could not be amplified, the giant pouched-rat (Cricetomys gambianus, subfamily Cricetomyinae) was also identified by $c y t b$ analyses (Fig. 2C). Although group III (divided into two subgroups, III-1 and III-2) was not identified by $C O I$ analysis (Fig. 2A), it is likely to be most related to the pygmy mouse (Mus minutoides, subfamily Murinae) by $c y t b$ analysis (Fig. 2C). On the other hand, the genus or species of groups VI and X could not be identified in this study. The numbers of animals captured in this study are shown in Table 2.

Serum samples of sufficient volume were chosen from each animal group and used as representative serum samples to determine the reactivity of HRP-conjugates. Serum samples of laboratory rat (Rattus norvegicus; Fig. 3I) and mouse (Mus musculus; Fig. 3J) were also included as the controls for HRP-conjugated anti-rat and anti-mouse antibodies, respectively. Serum samples of gerbil 2, rodent 1 (group X), rodent 3 (group III-2), rodent 5 (group VI) and dormouse were not tested, because of insufficient volume of serum samples. The HRP-conjugated anti-mouse, anti-rat, anti-Peromyscus and anti-hamster IgGs were highly reactive to antibodies of the multimammate rat (Fig. 3A) and the gerbil (Fig. 3C). Among 


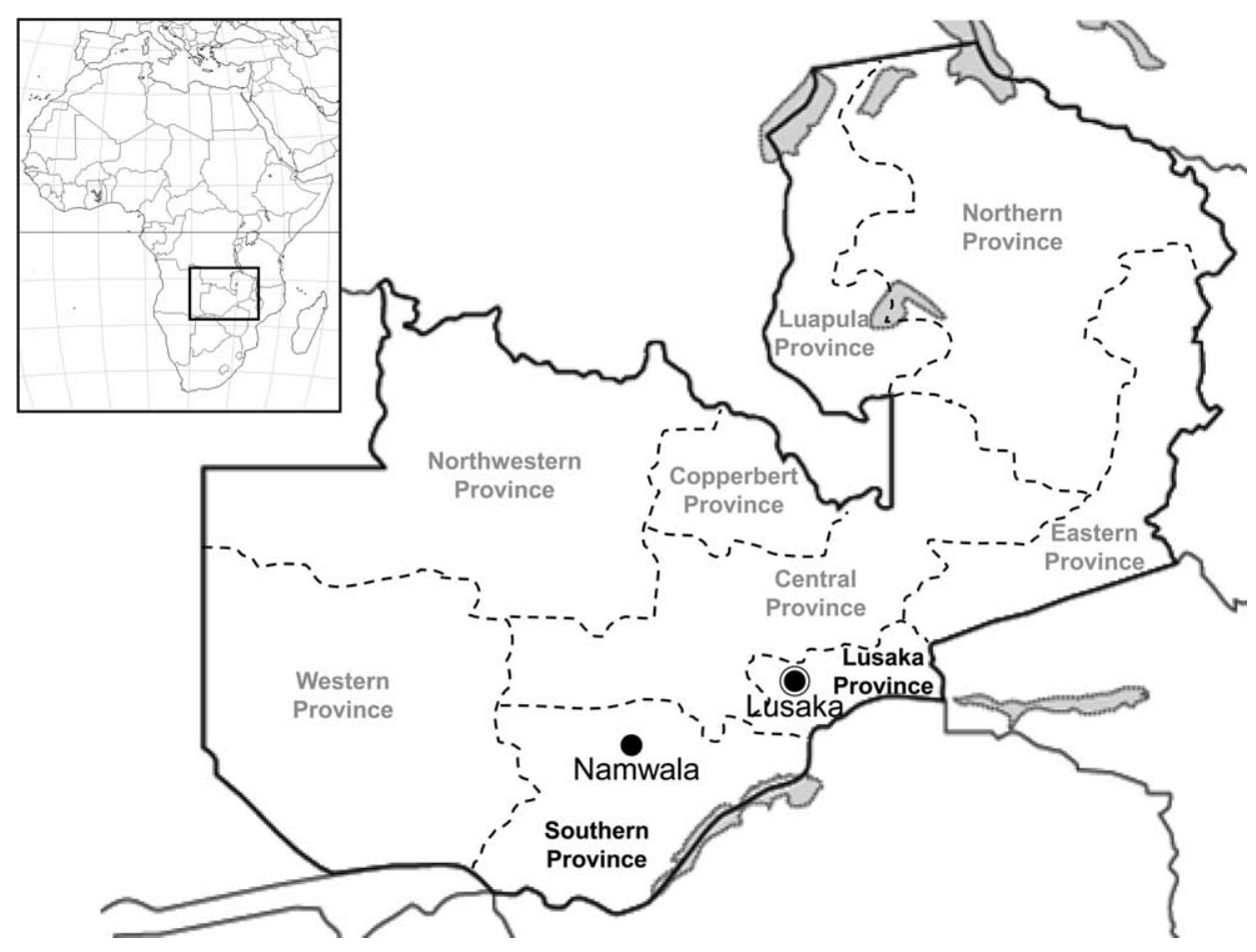

Fig. 1. Map of Zambia. Sampling sites, Lusaka and Namwala, are indicated.

them, HRP-conjugated anti-mouse antibody was most reactive, even if compared with the laboratory mouse (Fig. 3J), and its minimum effective concentration (MEC) was $0.03 \mu \mathrm{g} /$ $\mathrm{m} l$ to both species (black arrows in Fig. 3). HRP-conjugated anti-mouse IgG reacted with antibodies of the single-striped mouse (Fig. 3B), the pouched mouse (Fig. 3D) and the fat mouse (Fig. 3F), although not as strongly as with those of the multimammate rat (MEC of $0.5 \mu \mathrm{g} / \mathrm{ml}$; open arrows in Fig. 3). Their reaction profiles were distinguishable from that of the negative control (Fig. 3K), and they seemed like that of HRP-conjugated anti-mouse antibody against serum sample of the laboratory rat (Fig. 3I). No HRP-conjugates reacted with antibodies of the giant-pouched rat (Fig. 3E), rodent 2 (Fig. 3G) or rodent 4 (Fig. 3H).

By ELISA using cross-reactive secondary antibodies, preliminary examinations for several zoonoses, including plague, leptospirosis and hantavirus infection, were performed. HRP-conjugated anti-mouse IgG was used for serum samples of the multimammate rat and the gerbil 1 at the concentration of $0.05 \mu \mathrm{g} / \mathrm{m} l(\mathrm{MEC}$ of $0.03 \mu \mathrm{g} / \mathrm{m} l)$ and was used for serum samples of the pouched mouse, the single-striped mouse and the fat mouse at the concentration of $0.5 \mu \mathrm{g} / \mathrm{ml}$ (at MEC). For serum samples of the rat, HRPconjugated anti-rat IgG was used at the concentration of 0.75 $\mu \mathrm{g} / \mathrm{m} l$ (MEC less than $0.375 \mu \mathrm{g} / \mathrm{m} l$ [12]).

According to the criterion described above, two rodents (1 black rat and 1 multimammate rat) and one tanezumi rat were judged as positive to Yersinia pestis F1 and Leptospira rLipL32 antigen, respectively (Table 3). Five independent trials of PCR for plague pathogen could not detect any bac- terial gene from rodent liver DNA sample (data not shown). For hantavirus infection, a total of seven rodents were judged as positive for hantavirus antibody (Table 3). Of those seven rodents, four ( 2 multimammate rats, 1 fat mouse and 1 gerbil) were reactive to rNP103 of HTNV, and the other three (2 fat mice and 1 gerbil) were reactive to that of PUUV.

Correct classification of animals and selection of adequate secondary antibodies are crucial for serological examination of wild animals. In this study, a genetic approach was used for species identification of African wild rodents, followed by examination of various HRP-conjugates for their crossreactivity with antibodies of African rodents. Reactivity of HRP-conjugates with antibodies of various rodent species was investigated previously [12]. However, the African rodents captured and used in the present study were not included. Rodents captured in two regions of Zambia were grouped according to the phylogeny of COI (Fig. 2A), and each group was classified by phylogenetic analyses of $c y t b$. Since no sufficient reference data of $c y t b$ were available, species identification was not achieved for groups V, VI and IX. More precise data sets of $c y t b$ will be required for strict identification for African rodents in the subfamily Murinae, as well as for animals of the subfamilies Gerbillinae and Dendromurinae.

Applicability of commercially available HRP-conjugates was described previously [12], and in this study, we expanded the range of the test rodent species to African population captured in Zambia. Our results showed that HRP-conjugated anti-mouse $\mathrm{IgG}$ was more cross-reactive to heterologous IgGs than others tested (Fig. 3A, 3B, 3C, 3D and 3F). This 

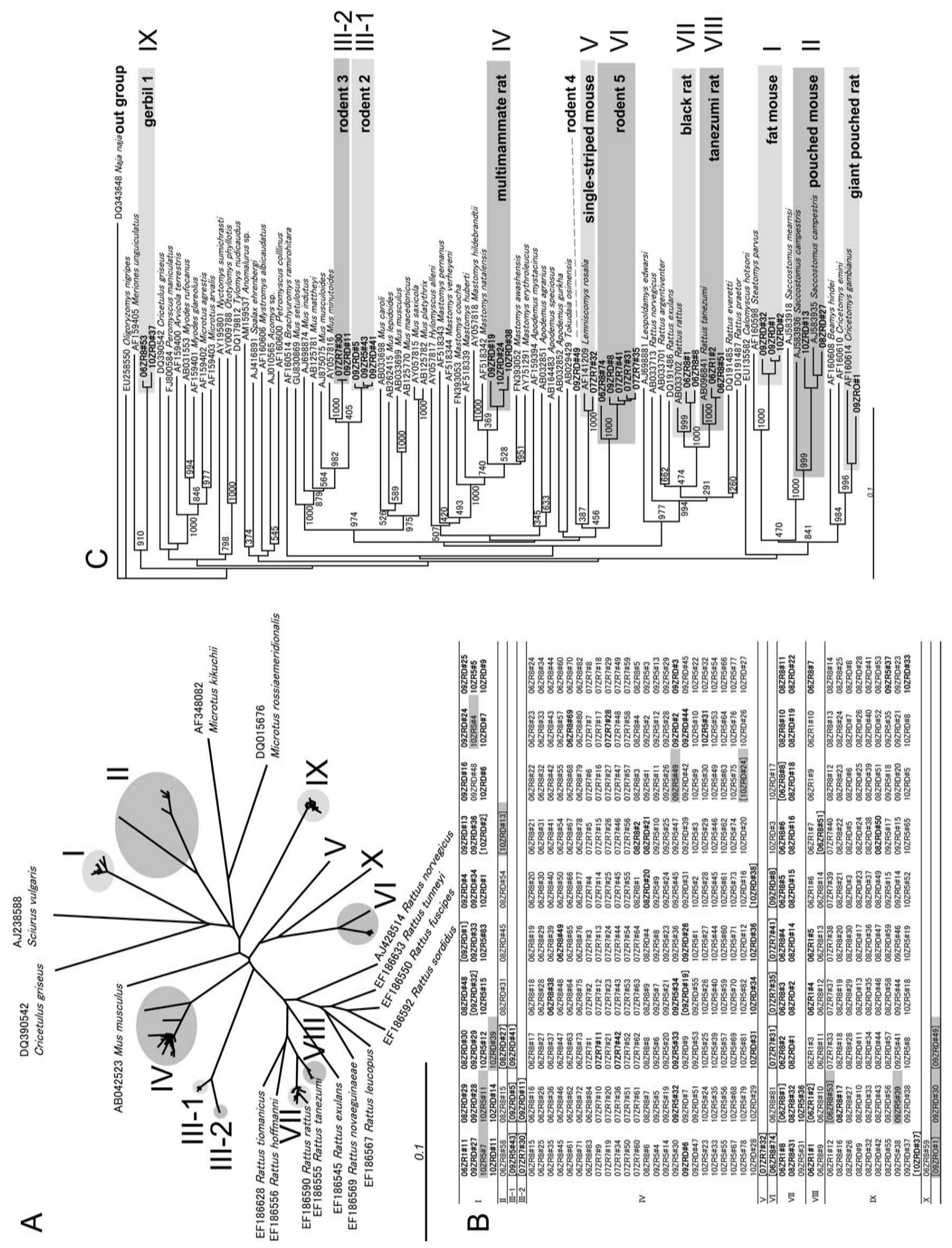
Table 2. Area and numbers of the animals captured in this study

\begin{tabular}{|c|c|c|c|c|c|c|c|c|c|c|c|c|c|}
\hline \multirow{2}{*}{$\begin{array}{l}\text { Area } \\
\text { Year }\end{array}$} & \multirow{2}{*}{ Subfamily } & & \multirow{2}{*}{$\begin{array}{l}\text { Scientific name } \\
\text { (tentative name) }\end{array}$} & \multicolumn{4}{|c|}{ Lusaka } & \multicolumn{4}{|c|}{ Namwala } & \multirow{2}{*}{ Total } & \multirow{2}{*}{ Group } \\
\hline & & & & 2006 & 2007 & 2008 & 2009 & 2007 & 2008 & 2009 & 2010 & & \\
\hline \multirow[t]{16}{*}{ Rodentia } & Murinae & Black rat & Rattus rattus & 8 & & 1 & & & 11 & 1 & 1 & 22 & VII \\
\hline & & Tanezumi rat & Rattus tanezumi & 17 & & & & & & & & 17 & VIII \\
\hline & & Multimammate rat & (Mastomys sp. IN-2012) & 62 & 50 & & & 3 & 12 & 53 & 83 & 263 & IV \\
\hline & & Single-striped mouse & (Lemniscomys sp. IN-2012) & & & & & 1 & & 1 & 1 & 3 & $\mathrm{~V}$ \\
\hline & & Rodent 1 & (Rodentia sp. IN-2012 (X)) & 1 & & & & & & & & 1 & $X$ \\
\hline & & Rodent 2 & (Rodentia sp. IN-2012 (III-1)) & & & & & 1 & & 1 & & 2 & III-1 \\
\hline & & Rodent 3 & (Rodentia sp. IN-2012 (III-2)) & & & & & & & 3 & & 3 & III-2 \\
\hline & & Rodent 4 & (Rodentia sp. 09ZRD\#49) & & & & & & & 1 & & 1 & - \\
\hline & & Rodent 5 & (Rodentia sp. IN-2012 (VI)) & 2 & & & & 3 & & 1 & 2 & 8 & VI \\
\hline & Gerbillinae & Gerbil 1 & (Gerbillinae sp. IN-2012) & 2 & & & & 5 & 57 & 20 & 10 & 94 & IX \\
\hline & & Gerbil 2 & & & & & & & & & 1 & 1 & $-^{* *}$ \\
\hline & Cricetomyinae & Pouched mouse & Saccostomus campestris & 1 & & & & & 5 & 2 & 3 & 11 & II \\
\hline & & Giant-pouced rat & Cricetomys gambianus & & & & 1 & & & & & 1 & - \\
\hline & Dendromurinae & Fat mouse & (Steatomys sp. IN-2012) & 1 & & & & & 3 & 17 & 17 & 38 & $\mathrm{I}$ \\
\hline & Graphiurinae & Dormouse & (Graphiurus sp. 09ZRD\#30) & & & & & & & 1 & & 1 & - \\
\hline & & & $\begin{array}{ll}\text { Total } \\
\end{array}$ & 94 & 50 & 1 & 1 & 13 & 88 & 101 & 118 & 466 & \\
\hline Soricomorpha & Crocidurinae & Shrew & & 1 & 0 & 0 & 0 & 0 & 0 & 3 & 2 & 6 & \\
\hline Afrotheria & Macroscelididae & Elephant shrew & & 1 & 1 & 0 & 0 & 0 & 0 & 0 & 0 & 2 & \\
\hline Others & & Lizard and Snake & & 0 & 0 & 0 & 0 & 0 & 1 & 1 & 0 & 2 & \\
\hline Total & & & & 96 & 51 & 1 & 1 & 13 & 89 & 105 & 120 & 476 & \\
\hline
\end{tabular}

*: Group is based on the phylogeny of COI. **: Not included in the phylogenetic analyses.

conjugate seems to be more reactive against antibody of the multimammate rat and the gerbil than that of the laboratory mouse (Mus musculus). The reaction profiles can be affected by the albumin/globulin ratio of the serum sample. Because wild rodents may develop immune response against natural antigens, it is considered that their serum samples may have lower albumin/globulin ratio compared to the laboratory animals. Since most of the rodent species captured in the present study were multimammate rats and gerbils (Table 2), HRP-conjugated anti-mouse IgG would be a useful detection tool in serological examination of the Zambian rodent population. Antibodies from the giant-pouched rat (Fig. 3E), and the rodents 2 and 4 (Fig. $3 \mathrm{G}$ and $3 \mathrm{H}$ ) reacted poorly with all of the conjugates tested. In contrast, antibodies of the multimammate rat and the gerbil reacted with anti-rat, anti-mouse, anti-Peromyscus and ant-hamster IgG (Fig. 3A and $3 \mathrm{C}$ ). Heterogeneous cross-reactivity of HRP-conjugates has been observed even within the same genus [12]. This reemphasizes the importance of proper classification of animals to be examined serologically and the importance of selection of appropriate detection techniques.
By using cross-reactive conjugates and optimized conditions for African rodents, ELISA used in this study showed the serological vestige of plague, leptospirosis and hantavirus infection (Table 3). Regarding the sero-surveillance, only the results of ELISA were reported. Although it is difficult to get definite diagnoses combined with other methods because of the limited volume of serum samples, the strict criterion employed in this study, that is, only outliers of the SmirnovGrubbs test being judged as positive, might have resulted in selection of true positives. This preliminary study will be followed by further investigations including highly sensitive sero-diagnosis, such as immunochromatography, and genetic detection of pathogens.

Although the detection rate of antibodies to the plague and leptospirosis was quite low, hantavirus infection seems to be endemic in the study area (Table 3). Seroprevalence described in this study can not be directly compared to the published results of serological studies on reservoir rodents $[1,9,16]$, because detection accuracy may be different. However, this is the first report of the existence of hantavirus in the eastern Africa. Taking account of the fact that several hantaviruses

Fig. 2. Phylogenetic analyses of COI and $c y t b$ sequences. A) Non-rooted phylogeny of 702 bp of $C O I$. The reference sequences employed are shown with accession numbers and scientific names. The individual numbers included in the analysis are listed in B) (white back-grounded). B) A list of individuals of the rodents analyzed. The individual number represents sampling year, country, kind of animal, sampling month (1-9, O, $\mathrm{N}, \mathrm{D})$ and serial number. Individuals analyzed by both $C O I$ and $c y t b$ are shown in bold letters, and those analyzed by only $c y t b$ are shown with a gray background. The representative individuals in C) are shown by brackets. C) A phylogenetic tree of $0.7 \mathrm{kbp}$ of $c y t b$. Only representative individuals and reference sequences are shown. Bootstrap values of major nodes are also shown. The individual numbers included in the analyses are listed in B) (bold or gray back-grounded). 


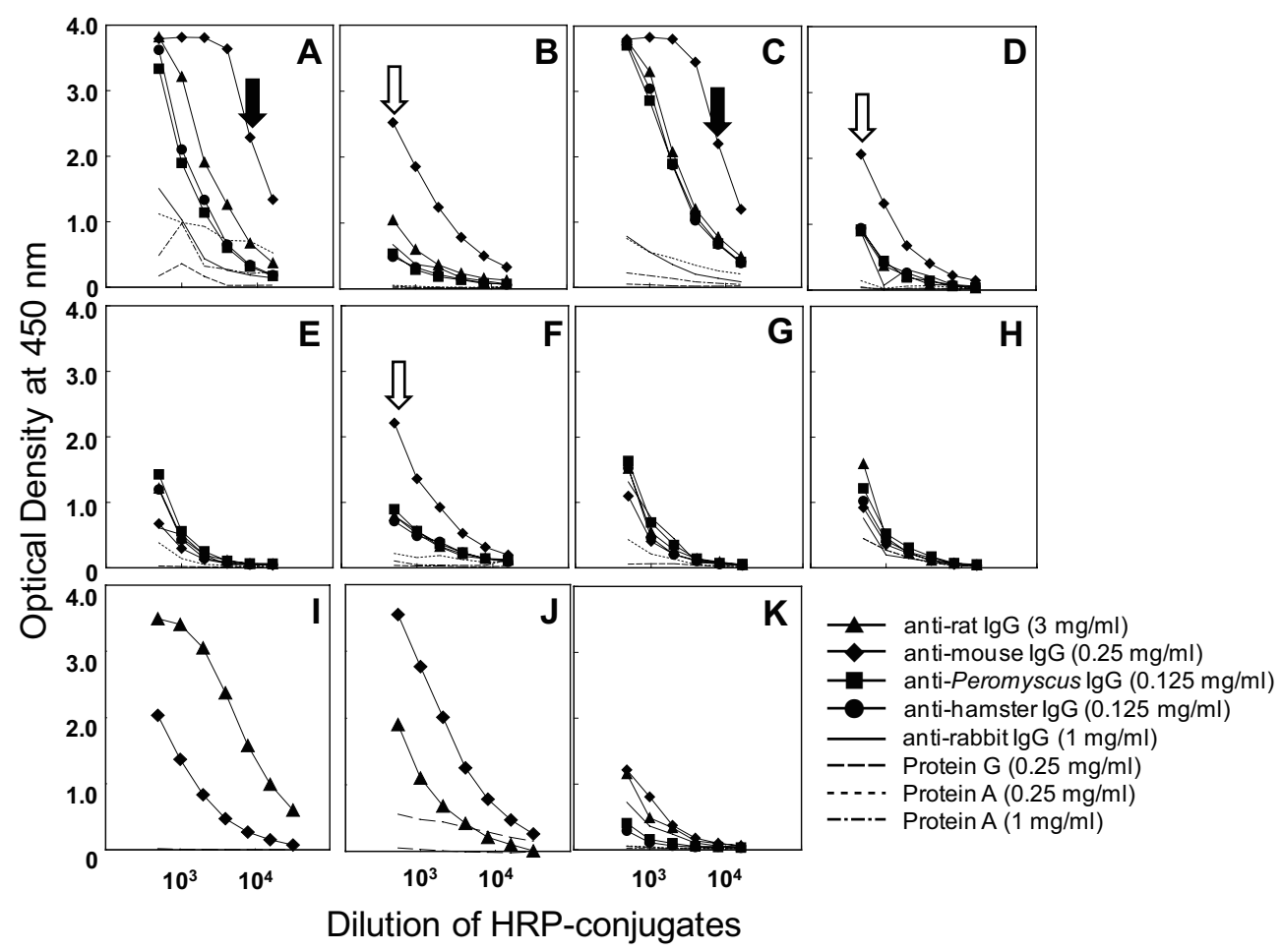

Fig. 3. Cross-reactivities of the HRP-conjugates to antibodies of heterologous rodents. Serially diluted HRPconjugates were examined against a constant volume of the serum samples in the wells of a plastic microplate. Concentrations of the stock solutions of HRP-conjugates are shown. Binding ability was assessed by measuring the color development of residential HRP activity. A: a multimammate rat (group IV: 10ZRD\#38), B: a single-striped mouse (group V: 10ZRD\#21), C: a gerbil (group IX: 10ZRD\#37), D: a pouched mouse (group II: 10ZRD\#13), E: a giant pouched rat (09ZRO\#1), F: a fat mouse (group I: 10ZRD\#39), G: rodent 2 (group III-1: 07ZR7\#30), H: rodent 4 (09ZRD\#49), I: a laboratory rat (Rattus norvegicus), J: a laboratory mouse (Mus musculus), K: negative control (no serum sample). Black and open arrows indicate minimal effective concentration (MEC).

Table 3. The number and prevalences of antibody positive animals for plague, leptospirosis and hantavirus infection

\begin{tabular}{|c|c|c|c|c|c|c|c|c|c|}
\hline \multirow{2}{*}{$\begin{array}{l}\text { Location } \\
\text { Year }\end{array}$} & & \multicolumn{3}{|c|}{ Lusaka } & \multicolumn{4}{|c|}{ Namwala } & \multirow{2}{*}{ Total } \\
\hline & & 2006 & 2007 & 2008 & 2007 & 2008 & 2009 & 2010 & \\
\hline No. tested & & 91 & 50 & 1 & 9 & 88 & 94 & 115 & 448 \\
\hline \multirow[t]{2}{*}{ Pest F1 } & No. positive & 1 & 0 & 0 & 0 & 0 & 0 & 1 & 2 \\
\hline & Positive (\%) & 1.09 & 0 & 0 & 0 & 0 & 0 & 0.87 & 0.45 \\
\hline \multirow[t]{2}{*}{ Leptospira rLipL32 } & No. positive & 1 & 0 & 0 & 0 & 0 & 0 & 0 & 1 \\
\hline & Positive (\%) & 1.09 & 0 & 0 & 0 & 0 & 0 & 0 & 0.22 \\
\hline \multirow[t]{2}{*}{ HTNV NP103 } & No. positive & 2 & 0 & 0 & 0 & 2 & 0 & 0 & 4 \\
\hline & Positive (\%) & 2.2 & 0 & 0 & 0 & 2.25 & 0 & 0 & 0.89 \\
\hline \multirow[t]{2}{*}{ PUUV NP103 } & No. positive & 0 & 0 & 0 & 0 & 1 & 0 & 2 & 3 \\
\hline & Positive (\%) & 0 & 0 & 0 & 0 & 1.12 & 0 & 1.74 & 0.67 \\
\hline
\end{tabular}

have been detected in the western part of Africa $[8,10,11$, 14, 17], hantavirus infections in Zambia, including human cases, may be noteworthy. In Africa, many rodent-related diseases have a major impact on public health. To control and prevent zoonoses, it is necessary to understand the true situation of local disease endemics. Sero-surveillance is one of the best methods to obtain informations of local disease endemics. Accordingly, wide-ranging and long-lasting serological surveillances should be conducted.

ACKNOWLEDGMENTS. This study was funded by the Program of Founding Research Centers for Emerging and Reemerging Infectious Diseases, Ministry of Education, Culture, Sports, Science and Technology (MEXT), Japan. 
Yersinia pestis antigens were kindly provided by Dr. Akihiro Hayashi, Yokohama Quarantine Station, Center for Inspection of Imported Foods and Infectious Diseases, Japan. The authors thank Mr. Ladslav Moonga, a laboratory assistant of HU-CZCZ, and Mr. Alick Chulu, a driver of HU-CZCZ, for their strong support in the field activities in Namwala. Mr. Mulenga, a laboratory assistant of the Veterinary School, UNZA also helped us to collect field samples in Namwala.

\section{REFERENCES}

1. Arellano, E., Castro-Arellano, I., Suzan, G., Gonzalez-Cozatl, F. X. and Jimenez, R. M. 2012. Antibody seroprevalence to hantaviruses in rodents from Reserva de la Sierra de Huautla, Morelos. West. North Am. Naturalist 72: 105-109.

2. Baker, E. E., Sommer, H., Foster, L. E., Meyer, E. and Meyer, K. F. 1952. Studies on immunization against plague. I. The isolation and characterization of the soluble antigen of Pasteurella pestis. J. Immunol. 68: 131-145. [Medline]

3. Corti, M. R., Castiglia, F. A. and Verheyen, W. 2004. Mitochondrial sequences and karyotypes reveal hidden diversity in African pouched mice (subfamily Cricetomyinae, genus Saccostomus). J. Zool. (Lond.) 262: 413-424. [CrossRef]

4. Flannery, B., Costa, D., Carvalho, F. P., Guerreiro, H., Matsunaga, J., Da Silva Domingos, E., Ferreira, A. G. P., Riley, L. W., Reis, M. G., Haake, D. A. and Ko, A. I. 2001. Evaluation of recombinant Leptospira antigen-based enzyme-linked immunosorbent assays for the serodiagnosis of leptospirosis. J. Clin. Microbiol. 39: 3303-3310. [Medline] [CrossRef]

5. Haake, D. A., Richard, G. C., Zuerner, R. L., Barnett, J. K., Barnett, D., Mazel, M., Matsunaga, J., Levett, P. N. and Bolin, C. A. 2000. The leptospiral major outer membrane protein LipL32 is a lipoprotein expressed during mammalian infection. Infect. Immun. 68: 2276-2285. [Medline] [CrossRef]

6. Hang'ombe, B. M., Nakamura, I., Samui, K. L., Kaile, D., Mweene, A. S., Kilonzo, B. S., Sawa, H., Sugimoto, C. and Wren, B. W. 2012. Evidence of Yersinia pestis DNA from fleas in an endemic plague area of Zambia. BMC Res. Notes 5: 72. [Medline] [CrossRef]

7. Irwin, D. M., Kocher, T. D. and Wilson, A. C. 1991. Evolution of the cytochrome $b$ gene of mammals. J. Mol. Evol. 32: 128-144. [Medline] [CrossRef]

8. Kang, H. J., Kadjo, B., Dubey, S., Jacquet, F. and Yanagihara, R. 2011. Molecular evolution of Azagny virus, a newfound hantavirus harbored by the West African pygmy shrew (Crocidura obscurior) in Cote d'Iboire. Virol. J. 8: 373. [Medline] [CrossRef]

9. Kariwa, H., Tkachenko, E. A., Morozov, V. G., Seto, T., Tani- kawa, Y., Kolominov, S. I., Belov, S. N., Nakamura, I., Hashimoto, N., Balkiev, A. E., Dzaguronova, T. K., Abu Daud, N. H., Miyashita, D., Medvedkina, O. A., Nakauchi, M., Ishizuka, M., Yoshii, K., Yoshimatsu, K., Arikawa, J. and Takashima, I. 2009. Epidemiological study of hantavirus infection in the Samara region of European Russia. J. Vet. Med. Sci. 71: 1569-1578. [Medline] [CrossRef]

10. Klempa, B., Fichet-Calvet, E., Lecompte, E., Auste, B., Aniskink, V., Meisel, H., Barriere, P., Koivogui, L., ter Meulen, J. and Kruger, D. H. 2007. Novel hantavirus sequences in shrew, Guinea. Emerg. Infect. Dis. 13: 520-522. [Medline] [CrossRef]

11. Klempa, B., Fichet-Calvet, E., Lecompte, E., Auste, B., Aniskink, V., Meisel, H., Denys, C., Koivogui, L., ter Meulen, J. and Kruger, D. H. 2006. Hantavirus in African wood mouse, Guinea. Emerg. Infect. Dis. 12: 838-840. [Medline] [CrossRef]

12. Lee, B. H., Yoshimatsu, K., Araki, K., Ogino, M., Okumura, M., Tsuchiya, K., Kariwa, H. and Arikawa, J. 2003. Detection of antibody for the serodiagnosis of hantavirus infection in different rodent species. Arch. Virol. 148: 1885-1897. [Medline] [CrossRef]

13. Robins, J. H., Hingston, M., Matisoo-Smith, E. and Ross, H. A. 2007. Identifying Rattus species using mitochondrial DNA. Mol. Ecol. Notes 7: 717-729. [CrossRef]

14. Sumibcay, L., Kadjo, B., Gu, S. H., Kang, H. J., Lim, B. K., Cook, J. A., Song, J. W. and Yanagihara, R. 2012. Divergent lineage of a novel hantavirus in the banana pipistrelle (Neoromicia nanus) in Cote d'Ivoire. Virol. J. 9: 34. [Medline] [CrossRef]

15. Tegshduuren, E., Yoshimatsu, K., Taruishi, M., Endo, R., Shimizu, K., Koma, T., Yasuda, S. P., Kariwa, H., Arikawa, J. and Ishihara, C. 2010. Different cross-reactivity of human and rodent sera to Tula virus and Puumala virus. Comp. Immunol. Microbiol. Infect. Dis. 33: e67-e73. [Medline] [CrossRef]

16. Truong, T. T., Yoshimatsu, K., Araki, K., Lee, B. H., Nakamura, I., Endo, R., Shimizu, K., Yasuda, S. P., Koma, T., Taruishi, M., Okumura, M., Truong, U. N. and Arikawa, J. 2009. Molecular epidemiological and serological studies of hantavirus infection in northern Vietnam. J. Vet. Med. Sci. 71: 1357-1363. [Medline] [CrossRef]

17. Weiss, S., Witkowski, P. T., Auste, B., Nowak, K., Weber, N., Fahr, J., Mombouli, J. V., Wolfe, N. D., Drexler, J. F., Drosten, C., Klempa, B., Leendertz, F. H. and Kruger, D. H. 2012. Hantavirus in Bats, Sierra Leone. Emerg. Infect. Dis. 18: 159-161. [Medline] [CrossRef]

18. Yoshimatsu, K., Arikawa, J., Tamura, M., Yoshida, R., Lundkvist, A., Niklasson, B., Kariwa, H. and Azuma, I. 1996. Characterization of the nucleocapsid protein of hantaan virus strain 76-118 using monoclonal antibodies. J. Gen. Virol. 77: 695-704. [Medline] [CrossRef] 